Revue d'histoire de l'Amérique française

DE REVUE D.HISTOIRE DE L'AMÉRIQUE FRANÇAISE

\title{
Introduction à la reconstitution de la population du Canada au XVII $^{\mathrm{e}}$ siècle \\ Étude critique des sources de la période 1665-1668
}

Hubert Charbonneau et Yolande Lavoie

Volume 24, numéro 4, mars 1971

URI : https://id.erudit.org/iderudit/303017ar

DOI : https://doi.org/10.7202/303017ar

Aller au sommaire du numéro

Éditeur(s)

Institut d'histoire de l'Amérique française

ISSN

0035-2357 (imprimé)

1492-1383 (numérique)

Découvrir la revue

Citer cet article

Charbonneau, H. \& Lavoie, Y. (1971). Introduction à la reconstitution de la population du Canada au XVII ${ }^{\mathrm{e}}$ siècle : étude critique des sources de la période 1665-1668. Revue d'histoire de l'Amérique française, 24(4), 485-511.

https://doi.org/10.7202/303017ar d'utilisation que vous pouvez consulter en ligne. 


\title{
INTRODUCTION A LA RECONSTITUTION DE LA POPULATION DU CANADA AU XVII ${ }^{\bullet}$ SIECLE
}

\author{
ETUDE CRITIQUE DES SOURCES \\ DE LA PERIODE 1665-1668
}

\author{
Hubert Charbonneau \\ Yolande Lavoie \\ Département de démographie \\ Université de Montréal
}

L'amélioration de nos connaissances dans les domaines relatifs à l'histoire de la population ou à la démographie historique n'est guère possible sans la critique approfondie des documents de base. Or, au Canada, nul ne s'est encore penché de façon systématique sur les faiblesses et les lacunes des principales sources démographiques anciennes. On ignore toujours si ce sont les recensements ou les registres paroissiaux qui pèchent le plus par défaut sous le Régime français. Cette insuffisance tient principalement à l'énormité de la tâche, puisque la population française du Canada aux XVII ${ }^{e}$ et XVIII ${ }^{e}$ siècles offre sans doute la documentation la plus complète qui existe sur une population de cette époque. La pléthore d'archives explique aussi pourquoi nous avons entrepris de reconstituer la population canadienne famille par famille, comme l'a fait Tanguay, et même individu par individu. Cette reconstitution est forcément limitée, par la qualité même des sources, au territoire actuel du Québec. ${ }^{1}$

$\mathrm{Au} \mathrm{XVII}^{\circ}$ siècle, on dispose de trois recensements nominatifs, ceux de 1666, 1667 et 1681 . Ceux-ci se révèlent d'autant plus précieux qu'il faut attendre le deuxième quart du XIX' siècle pour retrouver des documents comparables. D'autre part, l'implantation des faits d'enregistrement d'état civil par des prêtres

1 Voir à ce sujet: Hubert Charbonneau et Jacques Légaré, La démographie historique au Canada français avant 1800. Congrès général de l'Union internationale pour l'étude scientifique de la population (Londres, septembre 1969).

Hubert Charbonneau, Jacques Légaré, René Durocher, Gilles Paquet et Jean-Pierre Wallot, "La démographie historique au Canada", Recherches sociographiques, VIII, 2 (mai-août 1967).

[ 485 ]

RHAF, vol. 24, no 4 (mars 1971) 
déjà familiarisés en France avec la tenue des registres paroissiaux a permis d'accumuler, dès les origines du peuplement, une incomparable somme d'informations dont on a tiré des généalogies qui s'étendent aujourd'hui sur trois siècles et demi. Souvent perdus, ou avariés, les premiers registres paroissiaux ne sont certes pas parfaits. Plusieurs actes faits par des missionnaires sur des feuilles volantes n'ont jamais été retranscrits dans les registres et beaucoup de décès n'ont même jamais été enregistrés. On retrouve donc fréquemment la marque des difficultés d'implantation d'un excellent système administratif dans un rude milieu de colonisation.

Aussi, avant l'arrivée de Jean Talon, notre tentative de reconstitution se heurte-t-elle à d'innombrables difficultés, d'autant plus que les importants registres de l'Amirauté ont malheureusement disparu. Nous abandonnons donc volontiers la période antérieure aux premiers recensements nominatifs aux mains expertes des archivistes et des historiens. Et c'est pourquoi nous faisons débuter notre étude au ler janvier 1667.

Une telle entreprise ne pouvait cependant démarrer sans le recours à de longs et prudents essais. Nous avons rodé nos méthodes sur les sources de la période $1665-1668$, soit sur tous les actes des registres paroissiaux compris entre le 1er janvier 1665 et le 1er janvier 1669 de même que sur les deux recensements nominatifs de 1666 et de 1667 . Les résultats de notre étude pilote ont déjà paru ailleurs, ${ }^{2}$ mais nous croyons utile d'en présenter ici l'essentiel.

On trouvera ci-dessous une brève description des deux premiers recensements tels que les manuscrits nous les présentent. Cette description est fondamentale quand on envisage les étapes suivantes. Celles-ci consistent avant tout dans la comparaison des recensements entre eux, puis dans la confrontation de ces derniers avec les registres paroissiaux des années encadrantes. L'exploitation simultanée de ces deux sources, facilitée par le recours aux ordinateurs, aboutit à la correction réciproque des imperfections et des lacunes des données relatives à l'état de la population et de celles relatives au mouvement de la population.

2 Voir: Hubert Charbonneau et Jacques Légaré, "La population du Canada aux recensements de 1666 et 1667", Population, 22 (1967): 10311054.

Hubert Charbonneau, Jacques Légaré et Yolande Lavoie, "Recensements et registres paroissiaux du Canada durant la période de 1665-1668. Etude critique", Population, 25 (1970): 97-124. 


\section{LES DENOMBREMENTS NOMINATIFS DE 1666 ET 1667}

Les deux premiers recensements du Canada qui figurent parmi les premiers documents méritant vraiment ce nom dans les temps modernes, ont eu lieu au cours de la décennie qui a vu arriver le plus grand nombre d'immigrants au XVII' siècle. La population s'accroît alors rapidement et le nombre de personnes non encore "habituées" est relativement élevé. Arrivé avant l'état de 1666, le régiment de Carignan ne fut démembré qu'après l'état de 1667. Or les troupes ne sont pas inscrites dans ces documents. Il serait par ailleurs illusoire de croire que ces recensements ont été effectués sans erreurs ni omissions, la réalisation de ces opérations administratives constituant certes un défi dans les conditions de l'époque. ${ }^{3}$

\section{Effectifs et distribution géographique}

Ainsi qu'on peut le constater au tableau I, il n'est pas facile d'indiquer avec précision quels effectifs ont vraiment fait l'objet de ces deux dénombrements. Il y a lieu, croyons-nous, de clarifier une situation somme toute quelque peu complexe. Disons en premier lieu que les deux manuscrits contiennent des résultats chiffrés pour l'ensemble de la population et pour chaque région (colonne 1) : certains auteurs ont rejeté ces chiffres sans doute un peu précipitamment; rien en effet n'autorise à faire fi de ces données, si ce n'est qu'elles ne s'accordent pas avec le nombre d'individus nominativement recensés. Il est facile d'imaginer que les fonctionnaires de l'époque, à Québec, ont pu avoir le net sentiment que les agents recenseurs n'avaient pu atteindre tout le monde. La correspondance échangée entre Talon et Colbert prouve d'ailleurs que l'administration était consciente de ces lacunes, comme en font foi les propos suivants contenus dans une lettre que le ministre envoyait à Québec en date du 20 février 1668 : "J'ay esté surpris de ne trouver $\mathrm{d}^{3}$ le Rolle des habitans du Canada que $v^{\text {re }}$ Sec ${ }^{\text {re }}$ 'a remis es mains q. quatre mil trois cens douze personnes dont quinze cens soixante huict seul ${ }^{\text {mt }}$ sont en estat de porter les armes, et de ne trouver aussi que onze mil cens soixante quatorze arpens de terre cultivés sur q. $v^{\text {re }} \operatorname{Sec}^{\text {ro }}$ m'a dit quil y avoit beaucoup de fautes et d'obmission $d^{3}$ cet estat $p$. ce que $v^{s}$ 'y avez pu vacquer $v$. mesme a cause de vos maladies, $q^{1}$ connoissoit des familles entières qui n'y estoient $p$.

3 Les Amérindiens n'ont généralement pas été dénombrés hormis quelques femmes mariées à des Français, quelques pensionnaires chez les religieuses et quelques domestiques dont on ne connaît le plus souvent que le prénom. 
comprises et que $t^{3}$ les envoyés de l'année dernière soit homs soit filles et aussi les soldats qui ont pris des habitations ny sont pas mesme compris." 4

Le fait que le manuscrit ait été déposé à Paris a par ailleurs singulièrement compliqué la tâche des commentateurs du siècle dernier ainsi que nous l'apprend Sulte: "Voyez les recensements nominaux de la Nouvelle-France. Nous en avions copié trois à Paris, ceux des années $1666,1667,1681$. Le copiste ne comprenant pas les noms canadiens, les a massacrés. L'original serait facile à lire pour nous si nous l'avions." 5 Les copies déposées à Ottawa au moment du recensement de 1871 contenaient en effet de nombreuses erreurs de lecture et même des omissions. De surcroît, certains auteurs, comme Mgr Tanguay, se sont avisés de multiplier les revisions et corrections sans procéder de façon systématique. C'est pourquoi, vers 1935, P.-G. Roy a senti la nécessité de relire au moins le manuscrit de 1666 et de renouveler la publication imprimée de ce dernier document. ${ }^{6}$ Plus récemment, les Archives d'Ottawa ont obtenu le microfilm des originaux, ce qui n'a pas empêché la Socićté généalogique cana= dienne-française de publier l'état de 1667 d'après la version de Sulte une nouvelle fois corrigée. ${ }^{7}$ Nous sommes également tombés dans le même piège au début de nos travaux. Dans tous les cas, nul n'a jamais publié les manuscrits tels qu'ils se présentent et nul n'a effectué des corrections de façon systématique. Ainsi, au recensement de 1871, il est fait état d" "un double emploi de 21 noms, formant 5 familles" 8 , alors qu'il y a en fait 64 personnes inscrites deux fois au rôle de $1666 .^{9}$

Les colonnes 4 et 5 du tableau I, résultats de notre propre lecture, se rapportent aux effectifs véritablement inscrits sur les manuscrits, sans qu'aucune correction n'ait été apportée. Nous indiquons les résultats par région avec et sans les doubles

4 Rapport de l'Archiviste de la province de Québec (1930-1931): 94.

5 Benjamin Sulte, Mélanges historiques: études éparses et inédites. Editées par Gérard Malchelosse (Montréal, 1922), X: 24, in La Rivière-duLoup (en haut). 1-154.

- Rapport de l'Archiviste de la province de Québec (1935-1936):

7 Mémoires de la Société généalogique canadienne-française (janvieravril 1967), XVIII (1 et 2): 7-116.

8 Recensement de 1871, IV : 4.

${ }^{9}$ Les données du recensement de 1871 sont vraisemblablement le résultat des compilations de Cyprien Tanguay alors à l'emploi du Ministère de l'agriculture duquel dépendait la réalisation des recensements. 
TABLEAU I

Effectifs suivant la région aux recensements de 1666 et 1667 d'après divers compilateurs

\begin{tabular}{|c|c|c|c|c|c|c|c|c|c|c|}
\hline \multirow{3}{*}{ Régions } & \multicolumn{5}{|c|}{1666} & \multicolumn{5}{|c|}{1667} \\
\hline & \multirow{2}{*}{$\begin{array}{c}\text { Effectifs } \\
\text { inscrits } \\
\text { sur le } \\
\text { manus- } \\
\text { crit } \\
\\
\text { (1) }\end{array}$} & \multirow{2}{*}{$\begin{array}{c}\text { Effectifs } \\
\text { compilés } \\
\text { au recen- } \\
\text { sement } \\
\text { de } 1871 \\
\text { d'après } \\
\text { une copie } \\
\text { du ma- } \\
\text { nuscrit } \\
\text { (2) }\end{array}$} & \multirow{2}{*}{\begin{tabular}{|c|} 
Effectifs \\
réelle- \\
ment dé- \\
nombrés \\
d'après \\
le texte \\
imprimé \\
de \\
P.-G. Roy \\
(3)
\end{tabular}} & \multicolumn{2}{|c|}{$\begin{array}{l}\text { Effectifs } \\
\text { réellement } \\
\text { dénombrés } \\
\text { d'après le } \\
\text { microfilm du } \\
\text { manuscrit }\end{array}$} & \multirow[t]{2}{*}{$\begin{array}{l}\text { Effectifs } \\
\text { inscrits } \\
\text { sur le } \\
\text { manus- } \\
\text { crit }\end{array}$} & \multirow{2}{*}{$\begin{array}{l}\text { Effectifs } \\
\text { compilés } \\
\text { au recen- } \\
\text { sement } \\
\text { de } 1871 \\
\text { d'après } \\
\text { une copie } \\
\text { du ma- } \\
\text { nuscrit } \\
\text { (2) }\end{array}$} & \multirow{2}{*}{$\begin{array}{l}\text { Effectifs } \\
\text { réelle- } \\
\text { ment dé- } \\
\text { nombrés } \\
\text { d'après } \\
\text { le texte } \\
\text { imprimé } \\
\text { de } \\
\text { B. Sulte } \\
\text { (3) }\end{array}$} & \multicolumn{2}{|c|}{$\begin{array}{l}\text { Effectifs } \\
\text { réellement } \\
\text { dénombrés } \\
\text { d'après le } \\
\text { microfilm du } \\
\text { manuscrit }\end{array}$} \\
\hline & & & & $\begin{array}{c}\text { avec les } \\
\text { doubles } \\
\text { comptes } \\
(4)\end{array}$ & $\begin{array}{c}\text { sans les } \\
\text { doubles } \\
\text { comptes } \\
(5)\end{array}$ & & & & $\begin{array}{c}\text { avec les } \\
\text { doubles } \\
\text { comptes } \\
(4)\end{array}$ & $\begin{array}{c}\text { sans les } \\
\text { doubles } \\
\text { comptes } \\
(5)\end{array}$ \\
\hline Québec & 555 & 547 & 550 & 550 & 539 & 448 & 444 & 444 & 452 & 438 \\
\hline $\begin{array}{l}\text { Environs } \\
\text { de Québec }\end{array}$ & 663 & 590 & 589 & 589 & 583 & 1134 & 932 & 934 & 935 & 930 \\
\hline Ile d'Orléans & 471 & 452 & 472 & 472 & 440 & 529 & 426 & 426 & 427 & 426 \\
\hline $\begin{array}{l}\text { Côte de } \\
\text { Beaupré }\end{array}$ & 678 & 533 & 534 & 534 & 521 & 656 & 667 & 669 & 672 & 670 \\
\hline Côte du Sud & 6 & 13 & 13 & 13 & 13 & 113 & 114 & 112 & 113 & 111 \\
\hline $\begin{array}{l}\text { Trois-Rivières } \\
\text { et environs }\end{array}$ & 461 & 455 & 463 & 463 & 462 & 666 & 575 & 584 & 584 & 552 \\
\hline Montréal (île) & 584 & 625 & 625 & 625 & 624 & $*(766)$ & 760 & 765 & 765 & 750 \\
\hline TOTAL & 3418 & 3215 & 3246 & 3246 & 3182 & $*(4312)$ & 3918 & 3934 & 3948 & 3877 \\
\hline
\end{tabular}

* Les chiffres entre parenthèses n'apparaissent pas sur le document original, mais ont été extraits de la correspondance échangée entre Talon et Colbert. 
comptes. Nous espérons donc que les chiffres des colonnes 4 constituent une série définitive avant correction.

Si on se réfère aux colonnes 5 du tableau I, qui représentent en fait une première correction, on constate que la population augmente sensiblement d'un recensement à l'autre. L'augmentation porte sur toutes les régions sauf l'île d'Orléans où la diminution se révèle nettement suspecte. Dans le cas de Québec, la diminution des effectifs doit correspondre à des changements de définition de territoire, comme semble le prouver la forte augmentation de la population des environs de Québec. Nous reviendrons plus loin sur la critique des résultats.

\section{Structures suivant le sexe, l'âge et l'état matrimonial}

Il y a intérêt, croyons-nous, à analyser les résultats bruts tels que nous les révèlent les documents manuscrits. En premier lieu, parce que les individus recensés représentent vraisemblablement la grande majorité de la population; en second lieu, parce que c'est la seule démarche scientifique acceptable si on désire ensuite apprécier la vaieur et la nature des corrections.

\section{TABLEAU II}

Répartition de la population suivant le sexe et le groupe d'âges (pour 100 personnes au total)

\begin{tabular}{c||c|c|c||c|c|c}
\hline \multirow{2}{*}{$\begin{array}{c}\text { Groupe } \\
\text { d'âges }\end{array}$} & \multicolumn{3}{c||}{1666} & \multicolumn{3}{c}{1667} \\
\cline { 2 - 7 } & $\begin{array}{c}\text { Sexe } \\
\text { masculin }\end{array}$ & $\begin{array}{c}\text { Sexe } \\
\text { féminin }\end{array}$ & $\begin{array}{c}\text { Sexes } \\
\text { réunis }\end{array}$ & $\begin{array}{c}\text { Sexe } \\
\text { masculin }\end{array}$ & $\begin{array}{c}\text { Sexe } \\
\text { féminin }\end{array}$ & $\begin{array}{c}\text { Sexes } \\
\text { réunis }\end{array}$ \\
\hline $0-14$ & 21.0 & 19.0 & 40.0 & 21.3 & 19.9 & 41.2 \\
$15-29$ & 23.0 & 10.1 & 33.1 & 19.9 & 10.3 & 30.2 \\
$30-44$ & 13.7 & 5.5 & 19.2 & 13.6 & 5.9 & 19.5 \\
$45-59$ & 3.8 & 2.0 & 5.8 & 4.4 & 2.2 & 6.6 \\
60 et plus & 1.1 & 0.8 & 1.9 & 1.7 & 0.8 & 2.5 \\
Tous âges & 62.6 & 37.4 & 100.0 & 60.9 & 39.1 & 100.0 \\
\hline
\end{tabular}

Les non déclarés sont répartis au prorata.

La principale caractéristique de cette population de colons réside dans le déséquilibre entre les effectifs de chaque sexe. Dans un recensement comme dans l'autre, il y a plus d'hommes que de femmes à chaque âge (tableau II). Si on excepte les 
moins de 15 ans, nés pour la plupart au Canada, les effectifs masculins sont deux fois plus nombreux que ceux de l'autre sexe. Au total, il y a respectivement 170 et 156 hommes pour 100 femmes dans l'un et l'autre état. Cette disproportion est d'autant plus élevée qu'on est en droit de penser que ce sont surtout des hommes qui ont échappé au dénombrement. Une telle situation, qui résultait de l'immigration des dix années précédentes, "condamnait" au célibat une proportion d'hommes relativement importante et favorisait au premier chef la multiplication des coureurs de bois.

Un second trait singularise fortement cette population: il s'agit de l'opposition entre la population immigrée et celle née au pays. Ce dédoublement apparaît clairement sur la pyramide des âges (figure 1) et explique l'étranglement aux tranches d'âges de 10-14 et 15-19 ans. Cette population fraîchement installée affiche une extrême jeunesse puisque près des trois quarts des habitants ont moins de 30 ans et que plus de $90 \%$ sont âgés de moins de 45 ans.

La distribution des effectifs suivant l'état matrimonial est l'inéluctable conséquence du déséquilibre entre les sexes mentionné ci-dessus. D'une part, plus de la moitié des hommes de 20 ans et plus se déclarent célibataires, d'autre part, presque toutes les femmes de 15 ans et plus sont mariées, surtout si on exclut les religieuses.

Les deux états nominatifs fournissent aussi des renseignements sur les caractéristiques professionnelles de la population masculine. Plus que le métier exercé alors au Canada, ce sont surtout les qualifications des individus qu'on avait cherché à connaître, ainsi que le laisse entendre l'intendant Talon lui-même. Remarquons toutefois avec intérêt que presque tous les domestiques et engagés étaient célibataires alors que près de $85 \%$ des exploitants agricoles étaient mariés. ${ }^{10}$

\section{COMPARAISON DES RECENSEMENTS DE 1666 ET 1667}

A l'exemple de Colbert lui-même, il s'est trouvé de nombreux auteurs au cours des siècles pour dénoncer les lacunes de nos deux premiers dénombrements. Tel généalogiste a parfois souligné l'absence d'un ou de plusieurs individus, d'une ou de

${ }^{10}$ Le recensement de 1667 contient aussi des renseignements non démographiques (armes, bétail, arpents en valeur) que nous n'avons pas exploités. 
POPULATION INSCRITE SUR LES ÉTATS NOMINATIFS DE 1666 ET DE 1667 SUIVANT LE SEXE, ĹĀGe ET L'ÉTAT MATRIMONIAL
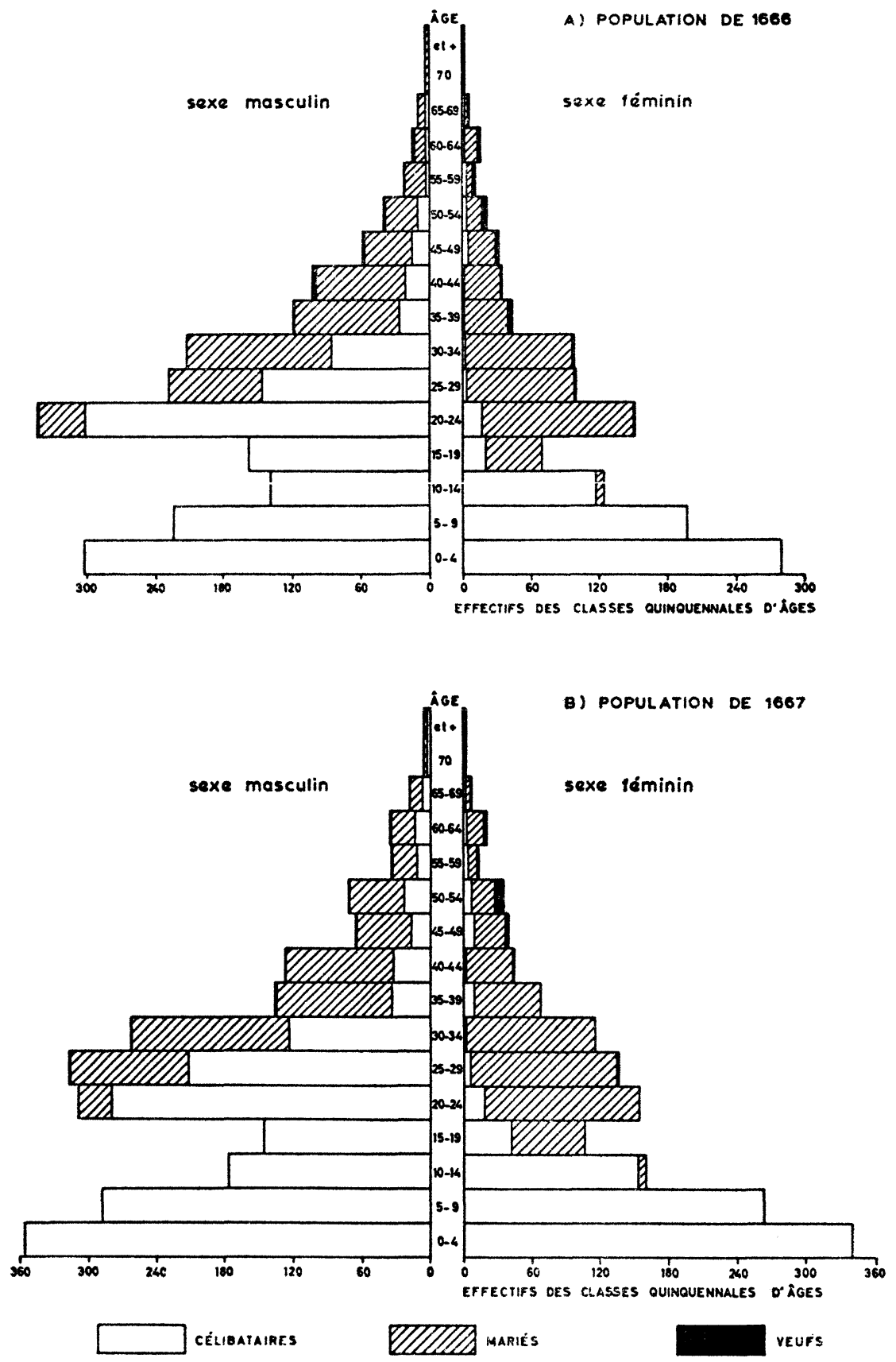

figure no1 
plusieurs familles, mais personne n'a encore effectué de critique à l'échelle de l'ensemble de la population. Pour notre part, nous nous proposons d'amorcer ici ce travail en opposant les deux recensements l'un à l'autre. Comme ils ne sont séparés que d'une année, il est d'ailleurs tentant de les comparer.

\section{Problèmes de jumelage de l'information}

La comparaison des deux recensements implique essentiellement la recherche des individus apparaissant dans l'une et l'autre liste, et aussi par conséquent des individus mentionnés dans une seule liste. Une telle opération suppose la mise en œuvre de moyens techniques relativement considérables. Grâce aux ordinateurs du Centre de calcul de l'Université de Montréal,11 nous disposions déjà d'une liste alphabétique de 7194 mentions d'individus recensés. Rappelons que toute mention d'individu, dans chaque dénombrement, a fait l'objet d'une carte perforée, conçue de façon à contenir outre les nom et prénom, le sexe, l'âge, l'état matrimonial, la profession, le lieu de résidence et le ménage auquel appartient l'individu recensé.

Cette laborieuse étape, par laquelle nous avons procédé au jumelage de cartes perforées intéressant une même personne, a dû être accomplie manuellement, en raison notamment des fréquentes variations orthographiques des noms. Le nom d'un même individu pouvait subir, d'un recensement à l'autre, des modifications d'ordre phonétique ou graphique, comme on peut en juger par les exemples suivants: on pouvait lire successivement Cadieu et Gadiou, Viville et Beville, Beloy et Peloy, Gomin et Gouin, Pournin et Pourvin, Amart et Aurat, mais aussi Chaleu et Chasseloup, Gaudry et Guidon, Demay et Dumers, Herlin et Allain. Tout en procédant avec assez de hardiesse, nous n'avons relié entre elles que les cartes réunissant une proportion raisonnable de caractéristiques analogues (liens de parenté, âge, lieu de résidence, par exemple).

A la fin du rapprochement des données, plusieurs catégories d'individus pouvaient être distinguées, comme en témoignent les rubriques du tableau III. On constate en effet que les 7194 cartes perforées mentionnées précédemment se rapportaient à 4470 individus différents. Seulement $58 \%$ de ces derniers apparaissent dans l'un et l'autre dénombrement. La proportion de cartes n'ayant pas fait l'objet de jumelage est donc assez élevée, mais on sait déjà qu'une fraction appréciable des 593 individus inscrits

11 Nous remercions vivement le Centre de calcul de l'Université de Montréal pour le concours indispensable de son personnel et de ses appareils. 
en 1666 seulement auraient dû être aussi recensés l'année suivante. L'importance des doubles comptes tient surtout au fait que les opérations de recensement s'étalaient sur plusieurs mois ainsi que nous le verrons plus loin.

\section{TABLEAU III}

Classification des individus inscrits à un recensement au moins

\begin{tabular}{l|r|r}
\hline \multicolumn{1}{c|}{ Catégorie d'individus } & $\begin{array}{c}\text { Nombre } \\
\text { d'individus }\end{array}$ & $\begin{array}{c}\text { Nombre de } \\
\text { cartes }\end{array}$ \\
\hline Inscrits aux deux recensements et mentionnés : & & \\
Une fois à chaque occasion & 2483 & 4966 \\
Une fois en 1666 et deux fois en 1667 & 48 & 144 \\
Deux fois en 1666 et une fois en 1667 & 55 & 165 \\
Deux fois à chaque occasion & 3 & 12 \\
Ensemble & 2583 & 5287 \\
Inscrits à un seul recensement et mentionnés : & & \\
En 1666 - une fois & 587 & 587 \\
- deux fois & 6 & 12 \\
En 1667 - une fois & 1268 & 1268 \\
- deux fois & 20 & 40 \\
Ensemble & 1881 & 1907 \\
$\quad$ Total & 4470 & 7194 \\
\hline
\end{tabular}

Caractéristiques des individus inscrits aux deux recensements

La comparaison des caractéristiques des personnes inscrites sur chacune des deux listes permet d'étudier le degré d'exactitude des déclarations faites aux agents recenseurs et aussi celui de la transcription subséquente. Par définition, les 2589 personnes ayant fait l'objet de jumelage sont entrées dans la population étudiée avant le premier dénombrement et n'en sont pas sorties avant le second. Ces personnes ont donc échappé aux risques de décès et d'émigration entre les deux recensements. Leurs principales caractéristiques apparaissent sur la figure 2 (pyramide a).

En comparant systématiquement l'orthographe des noms de chacun de ces individus d'un document à l'autre, on observe des variations plus ou moins importantes dans $55 \%$ des cas. On voit que la manière d'écrire un nom était loin d'être fixée. 
POPULATION RECENSÉE À UN SEUL OU AUX DEUX RECENSEMENTS SUIVANT LE SEXE. L'ÂGE ET LEETAT MATRIMONIAL
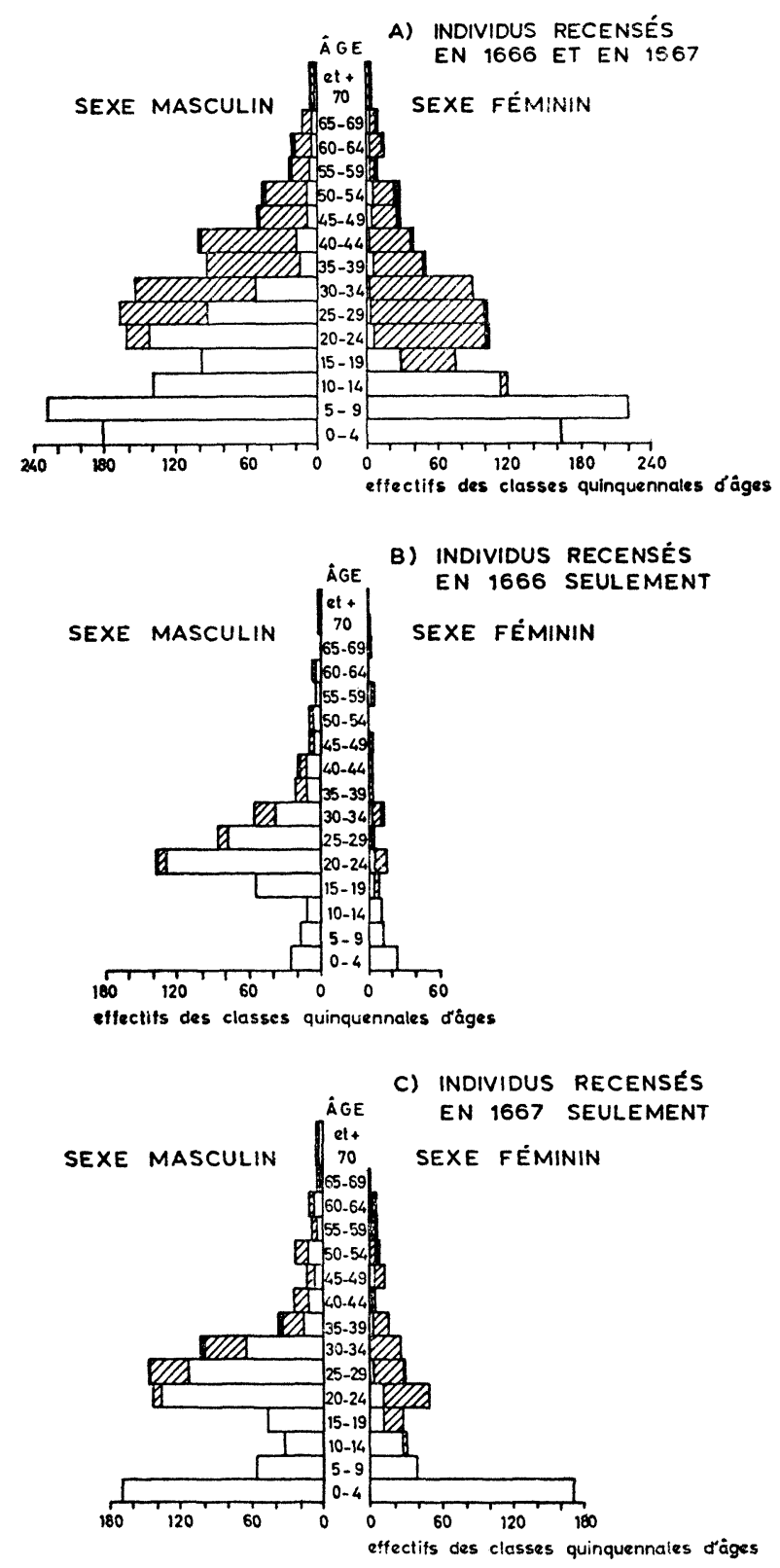
L'intérêt de la comparaison réside cependant dans l'examen des âges déclarés dans chacun des deux recensements. Comme ceux-ci portaient sur deux années consécutives, on a considéré comme normaux les cas où l'âge mentionné en 1667 était soit identique, soit supérieur de 1 ou 2 années à celui de 1666. Les écarts anormaux concernent $28 \%$ des individus. Cette dernière proportion, qui dépasse à peine $10 \%$ dans le groupe de moins de 15 ans, augmente graduellement avec l'âge jusqu'à atteindre $50 \%$ à 40 ans et plus. Les écarts de cinq années et plus affectent $9.0 \%$ des habitants de tous âges mais $26.5 \%$ des personnes de 40 ans et plus.

Des erreurs de raccordement et des erreurs de lecture ont pu causer une partie des écarts anormaux enregistrés. Ainsi, il est difficile d'expliquer que 29 individus n'aient pas le même sexe dans l'un et l'autre état.

\section{Caractéristiques des individus inscrits à un seul recensement}

Les 1881 personnes qui apparaissent à un seul recensement réunissent des caractéristiques spécifiques, ainsi que le fait ressortir la figure 2 (pyramides b et c). Le grand nombre d'individus ayant échappé au jumelage s'explique non seulement par le sous-enregistrement manifeste de l'un et l'autre recensement, mais aussi par les événements survenus au cours de la période intercensitaire: naissances (groupe $0-4$ ans de la pyramide c), décès et migrations. C'est pourquoi il $\mathrm{y}$ a deux fois plus de personnes inscrites à un seul recensement en 1667 qu'en 1666 . L'exploitation des registres paroissiaux va précisément nous permettre plus loin d'attribuer aux naissances et aux décès le rôle qui leur revient, le principal problème consistant à séparer la part des mouvements migratoires de celle du sous-dénombrement. Ce dernier facteur ne paraît sûrement pas négligeable lorsqu'on considère l'importance relative des hommes célibataires de 20-34 ans des pyramides $b$ et $\mathrm{c}$ de la figure 2 . La population de 15 ans et plus inscrite à un seul recensement compte d'ailleurs 7 fois plus d'hommes que de femmes en 1666 et 3 fois plus en 1667. Les individus non enregistrés par les agents recenseurs se rangent donc dans les catégories les plus susceptibles de migrer et de faire la traite des fourrures.

La proportion représentée par les personnes inscrites à un seul recensement varie considérablement d'une région à l'autre, aussi bien en 1666 qu'en 1667 (tableau IV). La ville de Québec, où arrivaient les immigrants, affiche les pourcentages les plus élevés. On peut déduire du même tableau que la Côte du Sud a 
TABLEAU IV

Population inscrite à un seul recensement par région

\begin{tabular}{|c|c|c|c|c|c|c|c|c|c|}
\hline \multirow[b]{2}{*}{ Région } & \multicolumn{4}{|c|}{ Recensement de 1666} & \multicolumn{5}{|c|}{ Recensement de 1667} \\
\hline & $\begin{array}{c}\text { Sexe } \\
\text { masculin }\end{array}$ & $\begin{array}{c}\text { Sexe } \\
\text { féminin }\end{array}$ & $\begin{array}{l}\text { Sexes } \\
\text { réunis }\end{array}$ & $\begin{array}{c}\text { Inscrits à un } \\
\text { seul recensement } \\
\text { pour } 100 \text { personnes } \\
\text { dénombrées }\end{array}$ & $\begin{array}{l}\text { Sexe } \\
\text { masculin }\end{array}$ & $\begin{array}{c}\text { Sexe } \\
\text { féminin }\end{array}$ & $\begin{array}{c}\text { Sexe non } \\
\text { déclaré }\end{array}$ & $\begin{array}{l}\text { Sexes } \\
\text { réunis }\end{array}$ & $\begin{array}{c}\text { Inscrits à un } \\
\text { seul recensement } \\
\text { pour } 100 \text { personnes } \\
\text { dénombrées }\end{array}$ \\
\hline Québec & 158 & 31 & 189 & 35.1 & 113 & 50 & - & 163 & 37.2 \\
\hline $\begin{array}{l}\text { Environs de } \\
\text { Québec }\end{array}$ & 91 & 16 & 107 & 18.4 & 235 & 127 & - & 362 & 38.9 \\
\hline Ile d'Orléans & 65 & 24 & 89 & 20.2 & 60 & 36 & - & 96 & 22.5 \\
\hline Côte de Beaupré & 30 & 11 & 41 & 7.9 & 132 & 56 & - & 188 & 28.1 \\
\hline Côte du Sud & 1 & - & 1 & 7.7 & 55 & 39 & 1 & 95 & 85.6 \\
\hline $\begin{array}{c}\text { Trois-Rivières } \\
\text { et environs }\end{array}$ & 87 & 15 & 102 & 22.1 & 104 & 50 & 37 & 191 & 34.6 \\
\hline Montréal (île) & 52 & 12 & 64 & 10.3 & 120 & 67 & 6 & 193 & 25.7 \\
\hline Total & 484 & 109 & 593 & 18.6 & 819 & 425 & 44 & 1288 & 33.2 \\
\hline
\end{tabular}


été extrêmement négligée par les agents recenseurs en 1666. Le nombre de femmes recensées en 1666 mais non en 1667 se révèle partout relativement faible.

\section{EXPLOITATION SIMULTANÉE DES RECENSEMENTS ET DE L'ETTAT CIVIL}

Dans un contexte d'enregistrement parfaitement exhaustif des faits d'état-civil, il serait relativement facile de déceler les lacunes d'un recensement surtout si les migrations extérieures ont peu d'importance. Or, dans le Canada du XVII' siècle, les mouvements migratoires se révèlent considérables alors que les registres paroissiaux n'ont pas la perfection souhaitée, ainsi qu'on le verra plus loin. Avant l'ordonnance du 7 novembre 1678, les registres ne furent tenus qu'en un seul exemplaire et sans l'intervention de l'autorité civile. La dispersion du peuplement le long du St-Laurent entraînait la circulation constante des missionnaires qui relevaient les actes sur des feuilles volantes, avant de les consigner dans les registres des principales paroisses.

\section{Méthode de dépouillement des registres paroissiaux}

Nous avons limité l'exploitation des registres paroissiaux à la période comprise entre le $1^{\text {er }}$ janvier 1665 et le $1^{\text {er }}$ janvier 1669, de façon à bien encadrer les deux premiers recensements nominatifs. Disséminées dans les plus anciennes paroisses de la colonies, les minutes se révèlent d'accès relativement difficile. Il convenait donc de les rassembler sous forme de microfilms, fidèles et peu encombrantes reproductions. C'est pourquoi nous avons microfilmé tous les registres antérieurs au $1^{\text {er }}$ janvier 1670. Il y avait alors une douzaine de paroisses et missions qui tenaient des registres, mais 9 d'entre elles seulement les ont conservés jusqu'à nos jours ; ${ }^{12}$ les premiers registres du Cap-de-la-Madeleine ont disparu et probablement aussi ceux de Champlain et du Sault-Saint-Louis (Caughnawaga).

Afin de tirer le plus grand profit possible des registres paroissiaux, on a d'abord constitué une fiche susceptible de recueillir presque toute l'information contenue dans ces documents (fig. 3). Cette fiche à modèle unique se divise en 3 parties:

12 Ce sont dans l'ordre chronologique: Notre-Dame-de-Québec, L'Immaculée-Conception des Trois-Rivières, Saint-Joseph de Sillery (registre déposé aux Archives de l'archevêché de Québec), Notre-Dame de Montréal, SainteAnne-de-Beaupré, La Visitation de Château-Richer (registre déposé au Petit Séminaire de Québec), Sainte-Famille de l'Ile d'Orléans, Sainte-Famille de Boucherville et mission du Lac St-Jean (registre déposé au Petit Séminaire de Québec). 
la première concerne les sujets d'acte, la seconde, les parents et conjoints de sujets d'acte et la troisième, les témoins et leurs conjoints. Pour tout individu mentionné dans un acte de baptême, mariage ou sépulture, qu'il soit signalé comme présent ou non, vivant ou décédé, on relève ses noms, prénoms et caractéristiques. Un code permettant l'exploitation mécanographique a été mis au point. Les individus paraissant dans un même acte sont reliés entre eux par l'identification de l'acte au haut de la fiche. L'identification est définie par le type d'acte et par les lieux et dates de l'événement ou de l'enregistrement de l'acte.

Pour l'ensemble des quatre années étudiées, nous avons dépouille 1276 actes d'état-civil, dont on a tiré 7252 cartes perforées correspondant à autant de noms inscrits dans les actes. L'information obtenue a été ensuite transcrite par les ordinateurs sous forme de listes alphabétiques et chronologiques et de tableaux à entrées multiples. Si on exclut les 68 actes relatifs aux Amérindiens, ${ }^{13}$ il ne reste que 1208 actes se rapportant à 768 naissances, 264 mariages et 176 décès. De prime abord, l'enregistrement des sépultures paraît nettement déficient, en dépit de la jeunesse de la population. Par ailleurs, le nombre considérable de mariages est lié à l'importante immigration de la période étudiée.

\section{Précisions sur les dates des recensements}

La confrontation mutuelle des sources nous a d'abord permis d'aboutir à un résultat inattendu, soit la détermination avec le maximum d'exactitude des périodes pendant lesquelles ont été exécutés les deux premiers dénombrements de la colonie. Historiens et généalogistes ont cru, à la suite de Tanguay, que le premier avait été dressé en février et mars 1666, le second en septembre et octobre $1667 .{ }^{14}$ En réalité les conclusions du réputé généalogiste à ce sujet sont d'autant plus discutables que ses procédés étaient rapides. Sa démonstration ne s'appuie que sur une quinzaine d'actes de naissance et laisse supposer que les opérations de recensement s'étaient déroulées simultanément dans toutes les régions en un laps de temps relativement court.

13 Nous ne retiendrons dans la reconstitution de la population que les Amérindiens vraiment intégrés au peuplement français. Les documents de l'époque ne permettent guère d'études démographiques sur les Amérindiens dont on ne connaît jamais la population de base. Par ailleurs, nous dépouillons les registres paroissiaux des missions indiennes, puisque les noms de nombreux Français y paraissent, ne serait-ce que comme témoins.

14 L'abbé Cyprien Tanguay, $A$ travers les registres (Montréal, Librairie St-Joseph, 1886), 51-52. Voir aussi: Recensement du Canada (1871), IV: 4 et 8 . 
acti $_{12}$ ND. MONTREAL

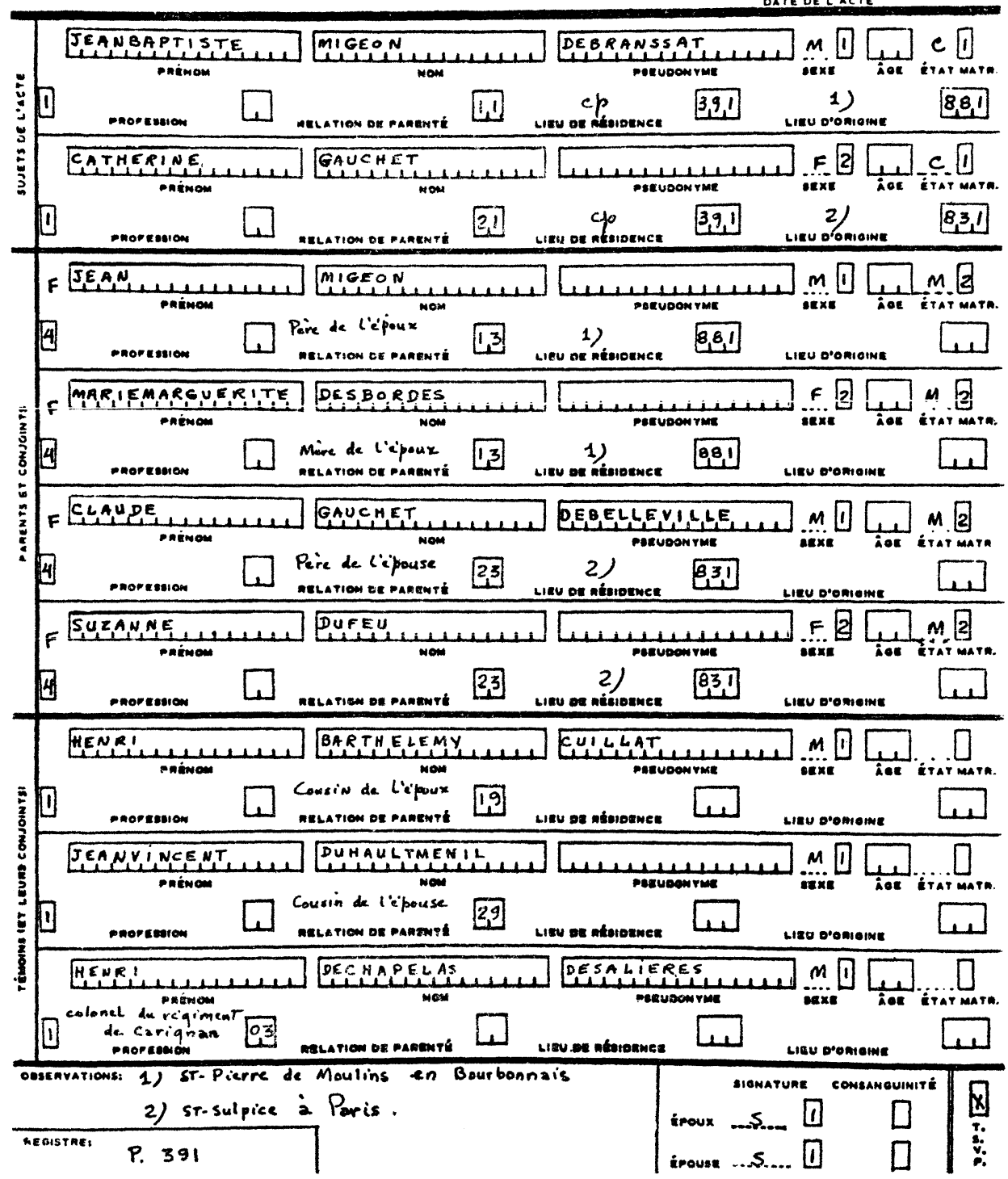


RECONSTITUTION DE LA POPULATION DU CANADA AU XVII0 SIECle 501

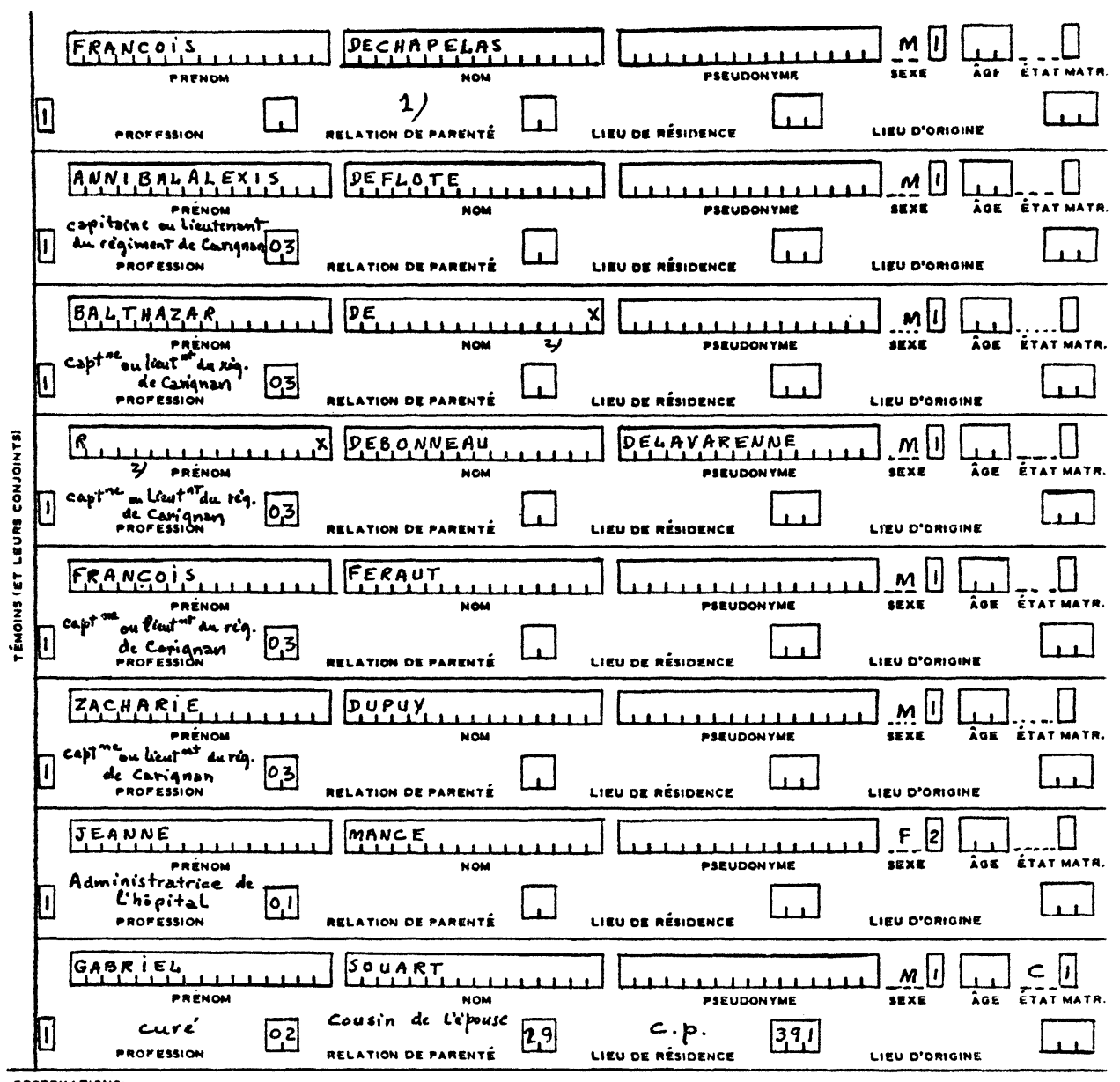

1) fils du précédent

2) illisible

Pour notre part, nous avons vérifié, dans chaque recensement, la présence de tous les sujets des actes de baptême et de sépulture des années 1666 et 1667, ainsi que l'état matrimonial des sujets des actes de mariage. On a pu ainsi déterminer, en considérant chaque région isolément, deux dates extrêmes entre lesquelles auraient été exécutées les opérations de recensement (figure 4). 
DÉROULEMENT DES OPÉRATIONS DE RECENSEMENT AU COURS DES ANNÉES 1666 ET 1667

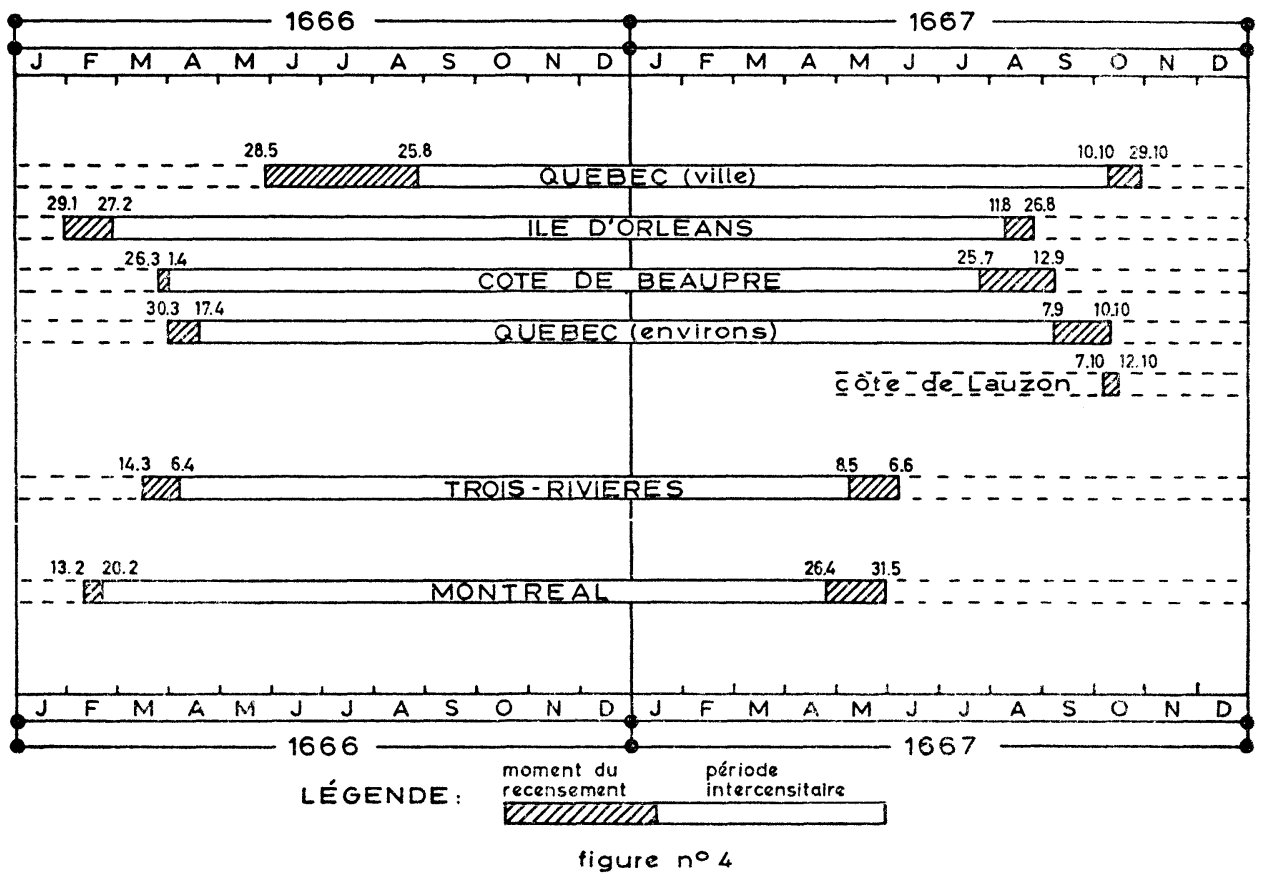

Nos résultats attestent que les agents recenseurs ne circulèrent pas partout en même temps dans l'ensemble du pays. Ce fait est d'ailleurs confirmé par l'intendant Talon, qui affirme avoir lui-même procédé au recensement de 1667 à Montréal, Trois-Rivières et dans les autres lieux en amont de Québec.15 Il s'ensuit que plusieurs mois d'été, soit l'équivalent d'une année d'immigration, séparent parfois deux régions à un même dénombrement. Hormis le cas de la ville de Québec en 1666, il semble que la collecte des données ait été accomplie assez rapidement dans le cadre d'une même région. En moyenne, les deux recensements se révèlent distants de 15 à 16 mois. L'intervalle atteint 18 mois à l'île d'Orléans, où deux années d'immigration séparent les deux états nominatifs.

15 Talon à Colbert, 27 octobre 1667, Rapport de l'Archiviste de la province de Québec (1930-1931): 80. 
Ainsi, à l'encontre de ce qu'on croyait jusqu'à présent, le dénombrement de 1666 n'a pas eu lieu exclusivement en février et mars, mais s'est plutôt déroulé entre le début de février et la fin d'août. On comprend dès lors pourquoi Talon n'a expédié "le rôle des familles" qu'en novembre de la même année. ${ }^{16}$ De même au second recensement, une demi-année s'est écoulée entre le début et la fin des opérations, de fin avril à fin octobre 1667 . Comme l'année précédente, les agents recenseurs passèrent à Montréal et aux Trois-Rivières au printemps, mais ils ne visitèrent le reste du pays que vers la fin de l'été ou même à l'automne. Le 25 août 1667, Talon écrivait d'ailleurs à Colbert: "Parce que présentement je me trouve fort indisposé, je remets encore au départ des autres vaisseaux qui sont en cette rade à vous envoyer le Rolle des habitants que vous recevrez fort exact." 17

\section{Sous-enregistrement des naissances et des décès}

Comme il y a complète indépendance entre les opérations de recensement et la tenue de l'état civil, le rapprochement des listes alphabétiques des deux sources nous permet d'estimer le sous-enregistrement des naissances et des décès. Les dénombrements contiennent par exemple les noms d'un certain nombre d'enfants dont l'acte de naissance ne nous est pas parvenu. En se limitant aux enfants nés entre le $1^{\text {er }}$ janvier 1665 et le recensement de 1667 , on a pu estimer à environ $12 \%$ le sousenregistrement des naissances pour cette période. Compte tenu de la disparition de certains registres, on est en droit de conclure que toutes les naissances étaient alors enregistrées, sauf celles suivies très rapidement d'un décès. Dans ce dernier cas, le risque de n'être pas recensé est évidemment très grand; tout comme la probabilité de n'apparaître dans quelque document que ce soit.

En ce qui concerne les décès, les lacunes de l'enregistrement ne tiennent pas seulement aux pertes de registres mais surtout aux omissions des rédacteurs des actes. Rappelons que même en France, l'enregistrement exhaustif des sépultures n'a généralement pas été atteint avant la déclaration royale du 9 avril 1736. On comprend d'ailleurs que les pouvoirs ecclésiastiques aient accordé moins d'importance à la consignation par écrit des décès qu'à celle des naissances ou des mariages. Les missionnaires venus de France au Canada ne se sentaient donc pas obligés d'inscrire les sépultures dans les registres et c'est pourquoi le

16 Talon à Colbert, 13 novembre 1666, ibid.: 58.

17 Talon à Colbert, 25 août 1667, ibid.: 75. 
sous-enregistrement des décès semble varier avec le sexe et l'âge et probablement aussi avec la profession et le lieu.

Pour les enfants nés entre le $1^{\text {er }}$ janvier 1665 et le $1^{\text {er }}$ janvier 1668 , on peut estimer à environ la moitié du total vraisemblable le nombre des décès de moins d'un an qui n'apparaissent pas dans les registres. Les documents de l'époque nous conduisent en effet à un taux de mortalité infantile de $102 \%$, valeur qui n'a été atteinte au Québec qu'après 1930. Pour le XVII ${ }^{e}$ siècle, un taux minimum compris entre 200 et $225 \%$ nous semble provisoirement plus acceptable.

Contrairement à ce qui se pratiquait alors en France, les prêtres inscrivaient mieux la mortalité infantile que celle des adultes. Durant la période comprise entre les deux recensements, 20 décès seulement seraient survenus parmi les 3182 individus réellement dénombrés en 1666, ce qui équivaudrait à un invraisemblable taux brut annuel de mortalité de l'ordre de $5 \%$. On ôtiendrait quâtre fois plus de décès dans une population où l'espérance de vie à la naissance serait d'environ 40 ans pour chaque sexe. Il s'ensuit qu'à l'époque de Talon, $15 \%$ seulement des décès féminins de plus d'un an auraient été enregistrés contre $35 \%$ des décès masculins. La mortalité des femmes était d'autant plus passée sous silence que les rédacteurs des actes s'attachaient particulièrement à inscrire les décès de type accidentel, comme ceux des noyés ou des personnes tuées par les Iroquois. C'est le cas notamment du premier registre de Montréal qui a provoqué tant de commentaires. On a là un bel exemple d'une réalité historique déformée par les sources: le fait qu'on ait surtout inscrit les décès des individus massacrés par les Amérindiens a conduit les commentateurs à exagérer considérablement l'importance relative de cette cause de décès.

\section{Sous-dénombrement des nouveau-nés}

En dépit de leurs lacunes, les registres paroissiaux peuvent servir à vérifier la qualité des recensements, d'autant plus que le risque d'être recensé au moins une fois est évidemment très grand dans le cas des individus constamment présents au cours des années 1666 et 1667 . C'est pourquoi nous nous sommes efforcés de rechercher ce que contenaient les deux dénombrements sur les enfants nés entre le $1^{\text {er }}$ janvier 1665 et l'état de 1666: suivant une hypothèse vraisemblable de mortalité, la proportion d'entre eux qui n'auraient pas été dénombrés en 1666 oscillerait autour de $20 \%$. En répétant les mêmes opérations pour les enfants nés entre les deux recensements, on constate que celui de 1667 se révèle plus exact que l'autre, car la proportion de 
nouveau-nés non déclarés n'y dépasserait pas $8 \%$. Si on réunit les deux recensements en une seule liste nominative, on en arrive à la conclusion que $3 \%$ seulement des enfants nés entre le $1^{\text {er }}$ janvier 1665 et le recensement de 1667 n'ont jamais été dénombrés, alors qu'ils devaient être encore vivants lors du passage des agents recenseurs. Pour ces générations nouvellement nées, l'erreur par défaut des registres paroissiaux, si faible soit-elle, se révèle quatre fois supérieure à celle des deux recensements réunis.

\section{Sous-dénombrement de l'ensemble des individus}

Dans le cas des adultes, leur absence dans les états nominatifs peut tenir au moins autant à une émigration temporaire qu'à une omission des agents recenseurs. Quoi qu'il en soit, excluons les personnes inscrites aux deux recensements et intéressons-nous à celles qui sont mentionnées à un seul recensement. Celles-ci peuvent être réparties suivant les catégories suivantes:

a) mentionnées dans un recensement et omises dans l'autre;

b) recensées en 1666 et sorties de la colonie par émigration ou décès avant le second recensement;

c) recensées en 1667 et entrées par naissance ou immigration depuis le premier recensement.

Il est évident que seules les personnes de la catégorie " $a$ " peuvent servir à établir l'importance du sous-dénombrement. Le recours aux registres paroissiaux de la seule période 1665-1668 nous a permis de retracer 427 omissions se répartissant de façon suivante (tableau V) :

a) 77 individus recensés en 1666 seulement mais apparaissant dans les registres entre le recensement de 1667 et le $1^{\text {er }}$ janvier 1669 ;

b) 350 individus recensés en 1667 seulement mais apparaissant dans les registres entre le $1^{\text {er }}$ janvier 1665 et le recensement de 1666.

D'autres individus n'apparaissent dans aucun dénombrement, mais pouvaient être présents à l'un ou même aux deux recensements. Ils appartiennent à l'une des catégories suivantes:

a) omis au recensement de 1666 et ayant quitté la colonie par émigration ou décès avant le second recensement;

b) omis au recensement de 1667 et étant entrés par naissance ou immigration depuis le recensement précédent;

c) omis dans les deux recensements. 
TABLEAU V

Individus oubliés à l'un ou aux deux recensements (présence prouvée par les registres de 1665-1668)

\begin{tabular}{|c|c|c|c|c|c|c|c|}
\hline \multirow[b]{2}{*}{ Région } & \multirow{2}{*}{$\begin{array}{l}\text { Recensés } \\
\text { en } 1667 \\
\text { et oubliés } \\
\text { en } 1666\end{array}$} & \multirow{2}{*}{$\begin{array}{l}\text { Recensés } \\
\text { en } 1666 \\
\text { et oubliés } \\
\text { en } 1667\end{array}$} & \multicolumn{3}{|c|}{ Jamais recensés } & \multirow{2}{*}{$\begin{array}{l}\text { Total des } \\
\text { individus } \\
\text { omis }\end{array}$} & \multirow{2}{*}{$\begin{array}{c}\text { Total } \\
\text { des } \\
\text { omissions }\end{array}$} \\
\hline & & & $\begin{array}{l}\text { oubliés } \\
\text { en } 1666 \\
\text { seulement }\end{array}$ & $\begin{array}{c}\text { oubliés } \\
\text { en } 1657 \\
\text { seulement }\end{array}$ & $\begin{array}{c}\text { oubliés } \\
\text { en } 1666 \\
\text { et en } 1667\end{array}$ & & \\
\hline Québec & 35 & 27 & 11 & 8 & 18 & 99 & 117 \\
\hline Environs de Québec & 125 & 20 & 2 & - & 3 & 150 & 153 \\
\hline Ile d'Orléans & 12 & 15 & - & 4 & - & 31 & 31 \\
\hline Côte de Beaupré & 66 & 4 & - & 1 & 4 & 75 & 79 \\
\hline Côte de Lauzon & 66 & - & - & - & - & 66 & 66 \\
\hline Trois-Rivières & 6 & 7 & - & 4 & 4 & 21 & 25 \\
\hline Montréal (île) & 40 & 4 & 1 & 4 & 4 & 53 & 57 \\
\hline Ensemble & 350 & 77 & 14 & 21 & 33 & 495 & 528 \\
\hline
\end{tabular}


La consultation des registres paroissiaux a permis de confirmer la présence à un ou même aux deux recensements de 101 personnes aucunement mentionnées par ceux-ci. Ces personnes peuvent être ainsi distribuées:

a) 14 individus oubliés au moins en 1666;

b) 21 individus oubliés au moins en 1667;

c) 33 individus oubliés aux deux recensements.

Ainsi, l'exploitation simultanée des deux recensements et de seulement quatre années de registres paroissiaux nous conduit à dénoncer 397 omissions en 1666 et 131 en 1667, soit un grand total de 528 omissions. On imagine facilement quels résultats on obtiendrait en exploitant les registres paroissiaux d'une période beaucoup plus longue.

Les données du tableau $\mathrm{V}$ font ressortir les lacunes des registres de la région des Trois-Rivières de même que la faiblesse du recensement de 1666 dans la région de la Côte du Sud. D'autre part, plusieurs individus retrouvés à l'aide des registres de Notre-Dame de Québec habitaient en réalité d'autres régions, notamment l'Ile d'Orléans et la Côte de Beaupré.

Ces dernières corrections, aussi provisoires soient-elles, modifient les résultats du tableau I (colonne 5) en majorant de facon appréciable les effectifs de toutes les régions du Canada de l'époque (tableau VI). Doit-on ajouter que ces dernières évaluations ne sont pas définitives. Les commentaires faits précédemment à l'examen du tableau I, à propos de Québec et de l'Ile d'Orléans, conservent leur pleine valeur.

\section{CONCLUSION}

Préalable à un vaste programme de recherches portant sur la reconstitution de la population du Canada, le présent article se situe à la fin d'un essai qui a duré quatre années. Au cours des pages qui précèdent, nous croyons avoir amorcé cette critique des sources si indispensable à l'étude de la population canadienne du passé.

Le principal enseignement qui se dégage de notre étude réside certes dans le fait que l'exploitation simultanée de sources, non pas fragmentaires mais imparfaites, peut conduire à une reconstitution presque parfaite de l'ensemble de la population. C'est du moins la conclusion que l'on peut dégager à propos du Canada sous le Régime français. Comme nous l'avions d'ail- 
TABLEAU VI

Effectifs corrigés, à l'aide des registres 1665-1668, suivant la région

\begin{tabular}{|c|c|c|c|c|c|c|c|c|c|}
\hline \multirow{3}{*}{ Région } & \multicolumn{3}{|c|}{1666} & \multicolumn{3}{|c|}{1667} & \multirow{3}{*}{$\begin{array}{l}\text { Taux } \\
\text { d'accrois- } \\
\text { sement } \\
\text { brut }\end{array}$} & \multirow{2}{*}{\multicolumn{2}{|c|}{$\begin{array}{l}\text { Dénombrés pour } \\
100 \text { présents } \\
\text { au total }\end{array}$}} \\
\hline & \multirow{2}{*}{$\begin{array}{c}\text { Dénom- } \\
\text { brés }\end{array}$} & \multirow{2}{*}{ Oubliés } & \multirow{2}{*}{ Présents } & \multirow{2}{*}{$\begin{array}{c}\text { Dénom- } \\
\text { brés }\end{array}$} & \multirow{2}{*}{ Oubliés } & \multirow{2}{*}{ Présents } & & & \\
\hline & & & & & & & & 1666 & 1667 \\
\hline Québec & 539 & 64 & 608 & 438 & 53 & 491 & -18.6 & 89.4 & 89.2 \\
\hline $\begin{array}{c}\text { Environs de } \\
\text { Québec }\end{array}$ & 583 & 130 & 713 & 930 & 23 & 953 & 33.7 & 81.8 & 97.6 \\
\hline Ile d'Orléans & 440 & 12 & 452 & 426 & 19 & 445 & -.15 & 97.3 & 95.7 \\
\hline Côte de Beaupré & 521 & 70 & 591 & 670 & 9 & 679 & 14.9 & 88.2 & 98.7 \\
\hline Côte de Lauzon & 13 & 66 & 79 & 111 & 0 & 111 & 40.5 & 16.4 & 100.0 \\
\hline Trois-Rivières & 462 & 10 & 472 & 552 & 15 & 567 & 20.1 & 97.9 & 97.4 \\
\hline Montréal (île) & 624 & 45 & 669 & 750 & 12 & 762 & 13.9 & 93.3 & 98.4 \\
\hline Ensemble & 3182 & 397 & 3579 & 3877 & 131 & 4008 & 12.0 & 88.9 & 96.7 \\
\hline
\end{tabular}


leurs présumé, les documents de base, recensements aussi bien qu'état civil, pèchent tous par défaut. Mais leur exploitation simultanée a démontré que la plupart des familles pouvaient être atteintes. Deux catégories d'individus échappent plus particulièrement à nos investigations : ce sont d'abord les enfants décédés très rapidement après leur naissance et surtout les jeunes hommes célibataires fréquemment éloignés de leur domicile par la traite des fourrures. Même dans le cas de ces derniers, un système d'enregistrement des décès bien tenu permettrait de les retrouver tôt ou tard à moins qu'ils aient définitivement quitté le Canada. Il semble toutefois que l'enregistrement des décès ait été nettement déficient avant que se fassent sentir les premiers effets de l'arrêt du Conseil Supérieur en date du 7 mai 1727. Fort heureusement, nous disposons pour remédier à ces lacunes de nombreux documents complémentaires: citons en particulier divers recensements, aveux et dénombrements, les registres de confirmations, les archives notariales se rapportant principalement à des contrats de mariage, à des contrats d'engagement pour les pays d'En-haut, à des testaments ou à des inventaires après décès.

Nous croyons donc fermement à la possibilité de réaliser un recensement pratiquement exhaustif, sinon chaque année, du moins tous les cinq ans, à partir du $1^{\text {er }}$ janvier 1667 , soit à la date qui nous paraît le mieux se prêter au début de ce genre d'opération. Dans cette perspective, nous avons entrepris le dépouillement exhaustif des registres paroissiaux du Canada du $\mathrm{XVII}^{\circ}$ siècle. Au début de l'année 1971 , le recensement de 1681 et tous les actes antérieurs à ce recensement avaient été dépouillés et transposés sur cartes perforées. A cet égard, l'intéressant parti que l'on peut tirer des ordinateurs électroniques autorise les plus grands espoirs. Dans ce type de recherches, s'ils permettent des gains de temps très appréciables, les ordinateurs ne font pas pour autant disparaître toutes les opérations manuelles, surtout en raison des innombrables variations orthographiques des noms. Ces dernières difficultés soulèvent tout le problème du jumelage automatique de l'information auquel nous nous sommes déjà résolument attaqués. 
TABLEAU ANNEXE

Recensement de 1666 - Population selon le sexe et l'ìtat matrimonial par année d'âge

\begin{tabular}{|c|c|c|c|c|c|c|c|c|c|c|c|c|c|c|c|}
\hline \multirow{2}{*}{ Age } & \multicolumn{5}{|c|}{ Sexe masculin } & \multicolumn{5}{|c|}{ Sexe féminin } & \multicolumn{5}{|c|}{ Sexes réunis } \\
\hline & Célib. & Mariés & Veufs & N.D. & $\begin{array}{c}\text { En- } \\
\text { semble }\end{array}$ & Célib. & Mariés & Teuves & N.D. & $\begin{array}{c}\text { En- } \\
\text { semble }\end{array}$ & Célib. & Mariés & Veufs & N.D. & $\begin{array}{c}\text { En- } \\
\text { semble }\end{array}$ \\
\hline $0-4$ & 303 & 0 & 0 & 0 & 303 & 279 & 0 & 0 & 0 & 279 & 582 & 0 & 0 & 0 & 582 \\
\hline $5-9$ & 223 & 0 & 0 & 0 & 223 & 197 & 0 & 0 & 0 & 197 & 420 & 0 & 0 & 0 & 420 \\
\hline $10-14$ & 138 & 0 & 0 & 0 & 138 & 117 & 7 & 0 & 0 & 124 & 255 & 7 & 0 & 0 & 262 \\
\hline $15-19$ & 157 & 0 & 0 & 0 & 157 & 20 & 49 & 0 & 0 & 69 & 177 & 49 & 0 & 0 & 226 \\
\hline $20-24$ & 301 & 41 & 1 & 0 & 343 & 17 & 134 & 1 & 0 & 152 & 318 & 175 & 2 & 0 & 495 \\
\hline $25-29$ & 145 & 82 & 0 & 0 & 227 & 3 & 94 & 2 & 0 & 99 & 148 & 176 & 2 & 0 & 326 \\
\hline $30-34$ & 85 & 127 & 0 & 0 & 212 & 2 & 94 & 2 & 0 & 98 & 87 & 221 & 2 & 0 & 310 \\
\hline $35-39$ & 26 & 92 & 1 & 0 & 119 & 1 & 38 & 4 & 0 & 43 & 27 & 130 & 5 & 0 & 162 \\
\hline $40-44$ & 18 & 80 & 4 & 0 & 102 & 1 & 31 & 2 & 0 & 34 & 19 & 111 & 6 & 0 & 136 \\
\hline $45-49$ & 15 & 42 & 1 & 0 & 58 & 4 & 25 & 2 & $\mathbf{0}$ & 31 & 19 & 67 & 3 & 0 & 89 \\
\hline $50-54$ & 11 & 28 & 1 & 0 & 40 & 3 & 14 & 4 & 0 & 21 & 14 & 42 & $\mathbf{5}$ & 0 & 61 \\
\hline $55-59$ & 5 & 16 & 1 & 0 & 22 & 3 & 5 & 3 & 0 & 11 & 8 & 21 & 4 & 0 & 33 \\
\hline $60-64$ & 3 & 9 & 2 & 0 & 14 & 1 & 12 & 3 & 0 & 16 & 4 & 21 & 5 & $\mathbf{0}$ & 30 \\
\hline $65-69$ & 2 & 8 & 0 & 0 & 10 & 1 & 2 & 2 & 0 & 5 & 3 & 10 & 2 & 0 & 15 \\
\hline $70-74$ & 1 & 1 & 0 & 0 & 2 & 0 & 1 & $\overline{1}$ & 0 & 2 & 1 & 2 & 1 & 0 & 4 \\
\hline $75-79$ & 0 & 5 & 1 & $\mathbf{0}$ & 6 & 0 & 0 & 1 & 0 & 1 & 0 & 5 & 2 & 0 & 7 \\
\hline $80-84$ & 1 & 1 & 1 & 0 & 3 & 0 & 0 & 0 & 0 & 0 & 1 & 1 & 1 & 0 & 3 \\
\hline $85-89$ & 0 & 1 & 0 & 0 & 1 & 0 & 0 & 0 & 0 & 0 & 0 & 1 & 0 & 0 & 1 \\
\hline 90 et + & 0 & 0 & 0 & 0 & 0 & 0 & 0 & 0 & 0 & 0 & 0 & 0 & 0 & 0 & 0 \\
\hline $\begin{array}{l}\text { N.D. } \\
\text { Tous }\end{array}$ & 66 & 0 & 0 & 0 & 66 & 17 & 0 & 1 & 0 & 18 & 83 & 0 & 1 & 0 & 84 \\
\hline $\begin{array}{l}\text { Tous } \\
\text { âges }\end{array}$ & 1500 & 533 & 13 & 0 & 2046 & 666 & 506 & 28 & 0 & 1200 & 2166 & 1039 & 41 & 0 & 3246 \\
\hline
\end{tabular}


TABLEAU ANNEXE

Recensement de 1667 - Population selon le sexe et l'état matrimonial par année d'âge

\begin{tabular}{|c|c|c|c|c|c|c|c|c|c|c|c|c|c|c|c|c|c|c|}
\hline \multirow[b]{2}{*}{ Age } & \multicolumn{5}{|c|}{ Sexe masculin } & \multicolumn{5}{|c|}{ Sexe féminin } & \multicolumn{3}{|c|}{ Non déclarés } & \multicolumn{5}{|c|}{ Sexes réunis } \\
\hline & Célib. & Mariés & Veufs & N.D. & $\begin{array}{c}\text { En- } \\
\text { semble }\end{array}$ & Célib. & Mariés & Veuves & N.D. & $\begin{array}{c}\text { En- } \\
\text { semble }\end{array}$ & Célib. & N.D. & $\begin{array}{c}\text { En- } \\
\text { semble }\end{array}$ & Célib. & Mariés & Veufs & N.D. & $\begin{array}{c}\text { En- } \\
\text { semble }\end{array}$ \\
\hline $0-4$ & 356 & 0 & 0 & 0 & 356 & 340 & 0 & 0 & 0 & 340 & 8 & 0 & 8 & 704 & 0 & 0 & 0 & 704 \\
\hline $5-9$ & 288 & 0 & 0 & 0 & 288 & 264 & 0 & 0 & $\mathbf{0}$ & 264 & 0 & 0 & 0 & 552 & 0 & 0 & 0 & 552 \\
\hline $10-14$ & 176 & 0 & 0 & 0 & 176 & 154 & 6 & 0 & 0 & 160 & 0 & 0 & 0 & 330 & 6 & 0 & 0 & 336 \\
\hline $15-19$ & 145 & 0 & 0 & 0 & 145 & 42 & 64 & 0 & 0 & 106 & 0 & 0 & 0 & 187 & 64 & 0 & 0 & 251 \\
\hline $20-24$ & 279 & 29 & 0 & 0 & 308 & 18 & 135 & 1 & 0 & 154 & 0 & 0 & 0 & 297 & 164 & 1 & 0 & 462 \\
\hline $25-29$ & 211 & 105 & 1 & 0 & 317 & 6 & 127 & 3 & 0 & 136 & 0 & 0 & 0 & 217 & 232 & 4 & 0 & 453 \\
\hline $30-34$ & 123 & 139 & 1 & 0 & 263 & 1 & 114 & 0 & 0 & 115 & 0 & 0 & 0 & 124 & 253 & 1 & 0 & 378 \\
\hline $35-39$ & 33 & 100 & 2 & 0 & 135 & 9 & 56 & 2 & 0 & 67 & 0 & 0 & 0 & 42 & 156 & 4 & 0 & 202 \\
\hline $40-44$ & 32 & 94 & 1 & 0 & 127 & 2 & 40 & 2 & 0 & 44 & 0 & 0 & 0 & 34 & 134 & 3 & 0 & 171 \\
\hline $45-49$ & 16 & 47 & 2 & 0 & 65 & 9 & 27 & 3 & 0 & 39 & 0 & 0 & 0 & 25 & 74 & 5 & 0 & 104 \\
\hline $50-54$ & 23 & 48 & 1 & 0 & 72 & 7 & 20 & 7 & 0 & 34 & 0 & 0 & 0 & 30 & 68 & 8 & 0 & 106 \\
\hline $55-59$ & 11 & 22 & 1 & 0 & 34 & 4 & 7 & 2 & 0 & 13 & 0 & 0 & 0 & 15 & 29 & 3 & 0 & 47 \\
\hline $60-64$ & 12 & 21 & 2 & 0 & 35 & 3 & 14 & 3 & 0 & 20 & 0 & 0 & 0 & 15 & 35 & 5 & 0 & 55 \\
\hline $65-69$ & 6 & 10 & 0 & 1 & 17 & 1 & 4 & 2 & 0 & 7 & 0 & 0 & 0 & 7 & 14 & 2 & 1 & 24 \\
\hline $70-74$ & 0 & 0 & 0 & 0 & 0 & 0 & 1 & 1 & 0 & 2 & 0 & 0 & 0 & 0 & 1 & 1 & 0 & 2 \\
\hline $75-79$ & 2 & 4 & 0 & 0 & 6 & 0 & 2 & 0 & 0 & 2 & 0 & 0 & 0 & 2 & 6 & 0 & 0 & 8 \\
\hline $80-84$ & 3 & 3 & 1 & 0 & 7 & 0 & 0 & 0 & 0 & 0 & 0 & 0 & 0 & 3 & 3 & 1 & 0 & 7 \\
\hline $85-89$ & 1 & 1 & 0 & 0 & 2 & 0 & 0 & 0 & 0 & 0 & 0 & 0 & 0 & 1 & 1 & 0 & 0 & 2 \\
\hline $90+$ & 0 & 0 & 0 & 0 & 0 & 0 & 0 & 0 & 0 & 0 & 0 & 0 & 0 & 0 & 0 & 0 & 0 & 0 \\
\hline N.D. & 10 & 2 & 0 & 2 & 14 & 6 & 7 & 0 & 0 & 13 & 2 & 55 & 57 & 18 & 9 & 0 & 57 & 84 \\
\hline $\begin{array}{l}\text { Tous } \\
\text { âges }\end{array}$ & 1727 & 625 & 12 & 3 & 2367 & 866 & 624 & 26 & 0 & 1516 & 10 & 55 & 65 & 2603 & 1249 & 38 & 58 & 3948 \\
\hline
\end{tabular}

